\title{
Facilitation of discriminated avoidance learning by dissociation of CS and manipulandum'
}

\author{
Gerald B. Biederman, M. R. D'Amato ${ }^{2}$ and Donald M. Keller \\ NEW YORK UNIVERSITY
}

\begin{abstract}
Many studies have reported inordinate difficulty in obtaining avoidance learning in discriminated bar-press and related situations, particularly with visual cues (illumination changes) serving as the CS. It is shown that avoidance learning can be substantially increased by dissociating the CS from the manipulandum, whichusually occur in close physical proximity. In verification of earlier work, discontinuous shock was again shown to enhance avoidance learning markedly.
\end{abstract}

\section{Problem}

A curious fact of discriminated avoidance learning is that, even under seemingly favorable conditions, it often does not occur. Rats given the simple task of pressing a bar (or turning a wheel) in the presence of a visual CS (change in illumination level) frequently fail to achieve even a modest level of avoidance learning despite extensive training (e. g., D'Amato \& Schiff, 1964; Meyer et al., 1960). Previous research aimed at uncovering the variables responsible for such failures has shown that discriminated avoidance learning can be greatly facilitated by the use of discontinuous shock, i. e., shock having brief on-periods and longer offperiods (D'Amato et al., in press). In the present study the contribution of another variable, location of the CS, was investigated. In virtually all bar-press avoidance studies employing a visual cue as the CS the stimulus source is located on the same wall as the bar, requiring $\mathrm{S}$ to approach the CS-a secondary aversive stimulusin order to make the instrumental response. Conceivably this is one of the factors which inhibits discriminated avoidance learning in the bar-press and related situations. A second purpose of the study was to determine whether discontinuous shock would continue to enhance avoidance learning when this presumably disrupting factor was 'removed.

\section{Method}

The Ss were 34 naive albino rats (13 males), 4-8 mos. of age, drawn from litters bred in our laboratory. Two standard Grason-Stadler Skinner boxes were modified by placing 3 identical 1-in. pilot lights on both the front and rear walls of the boxes, making it possible to program the CS (illumination of the pilot lights) to appear on either wall.

Group FC (N, 10) was trained with the CS located on the front wall and with conventional continuous shock as the US. Group RC (N, 14) was also trained with continuous shock, but the CS appeared on the rear wall. For
Group RD (N, 10) the CS was located on the rear wall, while the US was a discontinuous shock.

On Day 1 all Ss received 40 avoidance training trials, during which $\mathrm{S}$ was shaped on the escape response until a reasonable instrumental response appeared. Group RD received continuous shock during these trials. The CSUS interval was $5 \mathrm{sec}$; shock was maintained between .6 and $.8 \mathrm{ma}$; the intertrial interval, programmed by a film tape, averaged about $40 \mathrm{sec}$. When $\mathrm{S}$ failed to avoid, CS and US remained on until terminated by an escape response.

On Day 2, 500 training trials were given, the shock level being increased to .8-1.0 ma. During these trials Group RD experienced the discontinuous shock; the US began with a .2-sec. pulse of shock, followed by a 2.0-sec. off-period, the cycle being repeated until S made the instrumental response, which often fell into an offperiod.

Resuits

In Fig. 1 mean percentages of avoidances over the 500 trials of Day 2 are plotted in blocks of 50 trials. It is clear that under conventional training conditions (Group FC) only a very low level of avoidance is achieved by the end of the 500 trials. With the CS shifted to the rear wall, however, there occurs a substantial increase in avoidance performance (Group RC). That discontinuous shock enhances acquisition beyond the facilitation provided by the relocation of the CS is evident in the performance of Group RD.

A trend analysis of the first 200 trials of Day 2 (in blocks of 50 trials) produced significant between-groups effects ( F, 5.90; p <.01), and significant differences among the groups' linear trends (F, 10.31; p <.005). All pairwise comparisons of linear trends were significant at better than the .01 level. Analysis of the mean percentages of avoidances over the last 400 trials, an indicator of total avoidance behavior, showed Group RC to be superior to Group FC ( $p<.05)$, while the difference between Groups RC and RD did not achieve statistical significance $(t, 1.58)$.

\section{Diseussion}

It appears that the accidental physical association in most Skinner boxes of discriminandum and manipulandum accounts in part for the profound difficulty that rats have in acquiring a discriminated avoidance task employing visual cues. One need not look far for an explanation. In order to make the instrumental response $S$ must approach the highly directional $\mathrm{CS}$, which very early in 


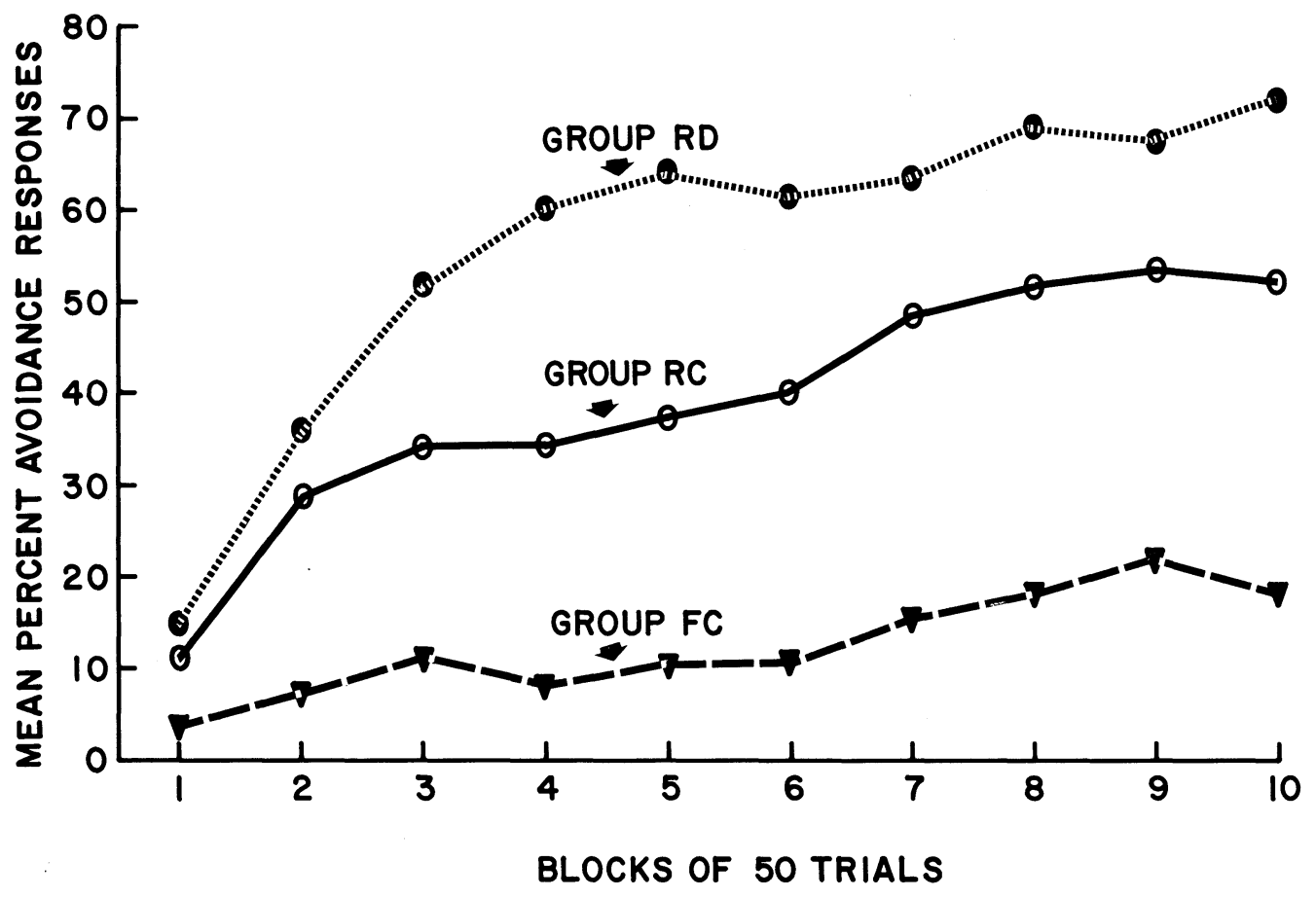

Fig. 1. Avoidance performance over the 500 trials of Day 2. Group FC was run under conventional training conditions, i. e., with the CS on the front wall and with continuous shock. Groups $\mathrm{RC}$ and $\mathrm{RD}$ were trained with the CS located on the rear wall and with continuous and discontinuous shock, respectively.

training becomes a secondary aversive cue. Thus the makings are ripe for a classical conflict situation. It is interesting that in similar experimental situations auditory stimuli, such as white noise, usually result in better avoidance learning than visual cues (e. g., D'Amato et al., in press). Rather than being indicative of a difference in underlying physiological mechanisms, this result may simply be due to the fact that auditory stimuli (which usually also emanate from the same area as the manipulandum) are less directional than visual stimuli. It might be noted in this connection that the average percentage of avoidances over the last 400 trials for Groups FC and RC combined, 32.0, is not far from the figure obtained for a group of Ss trained with a white noise CS but otherwise under very comparable conditions $-27.4 \%$ (D'Amato et al., in press).

Our interpretation of the facilitating effect of discontinuous shock is that it enhances generalization of the escape response to the preshock period. With continuous shock the escape response is by definition made in the presence of shock, the latter probably constituting the most salient stimulus component acting at the time the instrumental response is executed. By arranging things so that a substantial number of S's escape responses fall into off-periods, the shock component is largely removed from the stimulus complex associated with the instrumental response, and consequently generalization of the response to the preshock period is facilitated.
Judging from the performance of Group RD the facilitative effects of discontinuous shock and of dissociation of CS and manipulandum are not additive. Group RD averaged $63.7 \%$ avoidances over the last 400 trials, compared to $65.8 \%$ attained by 16 Ss of two other experiments run under highly comparable conditions except that the CS was located on the front wall. This suggests that, possibly because it encourages early emergence of avoidance responding, discontinuous shock generates considerably less conditioned aversiveness than continuous shock, with the result that there is much less reluctance on the part of S to approach the CS in its execution of the instrumental response.

\section{References}

D'AMATO, M. R., KELLER, D., \& DICARA, L. Facilitation of discriminated avoidance learning by discontinuous shock. J.comp. physiol. Psychol., in press.

D'AMATO, M. R., \& SCHIFF, D. Long-term discriminated avoidance performance in the rat. J.comp. physiol. Psychol., 1964, 57, 123-126.

MEYER, D. R., CHO, C., \& WESEMANN, A. F. On problems of conditioning discriminated lever-press. avoidance responses. Psychol. Rev., 1960, 67, 224-228.

\section{Notes}

1. This work was supported by Grant GB-1515 from the National Science Foundation.

2. Now at Rutgers University, New Brunswick, New Jersey. 